\title{
Application of Novel Intraoperative Neuromonitoring System Using an Endotracheal Tube With Pressure Sensor During Thyroid Surgery: A Porcine Model Study
}

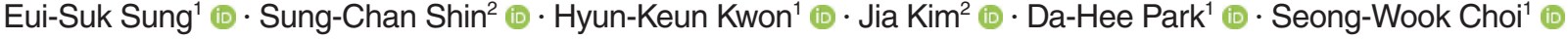 \\ Sang-Hoon $\mathrm{Kim}^{2}$ (i) $\cdot$ Jin-Choon Lee ${ }^{1}$ (i) $\cdot$ Jung-Hoon Ro ${ }^{3}$ (i) $\cdot$ Byung-Joo Lee $^{2}$ (i) \\ ${ }^{I}$ Department of Otolaryngology-Head and Neck Surgery, School of Medicine, Pusan National University and Research Institute for Convergence \\ of Biomedical Science and Technology, Pusan National University Yangsan Hospital, Yangsan; ${ }^{2}$ Department of Otorhinolaryngology-Head and \\ Neck Surgery, School of Medicine, Pusan National University and Medical Research Institute, Pusan National University Hospital, Busan; \\ ${ }^{3}$ Department of Biomedical Engineering, Pusan National University Hospital, Busan, Korea
}

Objectives. The loss of signal during intraoperative neuromonitoring (IONM) using electromyography (EMG) in thyroidectomy is one of the biggest problems. We have developed a novel IONM system with an endotracheal tube (ETT) with an attached pressure sensor instead of EMG to detect laryngeal twitching. The aim of the present study was to investigate the feasibility and reliability of this novel IONM system using an ETT with pressure sensor during thyroidectomy in a porcine model.

Methods. We developed an ETT-attached pressure sensor that uses the piezoelectric effect to measure laryngeal muscle twitching. Stimulus thresholds, amplitude, and latency of laryngeal twitching evaluated using the pressure sensor were compared to those measured using transcartilage needle EMG. The measured amplitude changes by EMG and the pressure sensor during recurrent laryngeal nerve (RLN) traction injury were compared.

Results. No significant differences in stimulus threshold intensity between EMG and the pressure sensor were observed. The EMG amplitude detected at $0.3 \mathrm{~mA}$, increased with increasing stimulus intensity. When the stimulus was more than $1.0 \mathrm{~mA}$, the amplitude showed a plateau. In a RLN traction injury experiment, the EMG amplitude did not recover even 20 minutes after stopping RLN traction. However, the pressure sensor showed a mostly recovery.

Conclusion. The change in amplitude due to stimulation of the pressure sensor showed a pattern similar to EMG. Pressure sensors can be feasibly and reliably used for RLN traction injury prediction, RLN identification, and preservation through the detection of laryngeal muscle twitching. Our novel IONM system that uses an ETT with an attached pressure sensor to measure the change of surface pressure can be an alternative to EMG in the future.

Keywords. Intraoperative Neurophysiological Monitoring; Pressure Sensor; Electromyography; Recurrent Laryngeal Nerve; Thyroidectomy

\footnotetext{
- Received August 7, 2019

Revised October 27, 2019

Accepted November 13, 2019

- Corresponding author: Byung-Joo Lee

Department of Otorhinolaryngology-Head and Neck Surgery, School of Medicine, Pusan National University and Medical Research Institute, Pusan National University Hospital, 179 Gudeok-ro, Seo-gu, Busan 49241, Korea Tel: +82-51-240-7675, Fax: +82-51-246-8668

E-mail: voiceleebj@gmail.com
}

\footnotetext{
- Co-Corresponding author: Jung-Hoon Ro

Department of Biomedical Engineering, Medical Research Institute, Pusan

National University Hospital, School of Medicine, Pusan National

University, 179 Gudeok-ro, Seo-gu, Busan 49241, Korea

Tel: +82-51-240-7933, Fax: +82-51-240-7564

E-mail: jhro@pnu.edu
}

Copyright $\odot 2020$ by Korean Society of Otorhinolaryngology-Head and Neck Surgery

This is an open-access article distributed under the terms of the Creative Commons Attribution Non-Commercial License (https://creativecommons.org/licenses/by-nc/4.0)

which permits unrestricted non-commercial use, distribution, and reproduction in any medium, provided the original work is properly cited. 


\section{INTRODUCTION}

Recurrent laryngeal nerve (RLN) palsy after thyroidectomy affects patients' quality of life and may cause legal problems [1,2]. Therefore, preservation of the RLN during thyroid surgery is of the utmost importance. However, it is known that vocal fold palsy (VFP) occurs in $2.3 \%$ to $26 \%$ of patients after thyroid surgery [3]. The average incidence of VFP after thyroidectomy was $9.8 \%$ [3]. The gold standard method for RLN preservation is visual identification [4-6]. However, visual identification cannot confirm the functional integrity of the nerve, but only the structural integrity, and thus, nerve damage may remain undetected. To address these issues, intraoperative neuromonitoring (IONM) was introduced during thyroid surgery [7].

Conventional IONM is comprised of stimulation side and recording side. Recently, several new devices have been reported to overcome the disadvantages of IONM stimulation side [8-10]; moreover, several limitations of IONM recording side when performing electromyography (EMG) on the vocalis muscle, have been reported. First, it was reported that malposition of endotracheal surface electrodes occurs in $3.8 \%$ to $23 \%$ of the patients [11-13]. If one of the two electrodes is not in contact with the vocal cords, loss of signal (LOS) is likely to occur and it becomes difficult to monitor EMG adequately [7,14]. It is reported that about $10 \%$ of patients with thyroid surgery need the position of the endotracheal tube (ETT) adjusted during surgery owing to electrode-vocal cord contact failure [15]. In addition, detection of EMG signal is poor when saliva or sputum is present. As a result, sensitivity is low and positive predictive value varies from $10 \%$ to $92 \%$ during thyroid surgery under IONM [16].To resolve the limitations of recording side, several attempts have been made, such as transcartilage needle EMG, transcartilage surface EMG, transcutaneous surface EMG, and postcricoid surface EMG to overcome the disadvantages of conventional EMG ETT electrodes [17-23]. The gold standard for evaluating the RLN status when LOS occurs is the palpation of laryngeal muscle twitch using a finger [7]. RLN injuries such as inadvertent transection, entrapment, and clamping have been rarely reported since the introduction of IONM, but traction injury caused

\section{H I G G H L I G G H T S}

- The loss of signal during neuromonitoring using electromyography in thyroidectomy is a pressing issue.

- Intraoperative neuromonitoring (IONM)-based laryngeal muscle twitching detection using endotracheal tube (ETT) attached pressure sensor.

- Amplitude changes depending on stimulation in pressure sensor show patterns similar to electromyography.

- The application of IONM using an ETT with a pressure sensor during surgery is feasible and reliable. by thyroid medial retraction (a common mechanism of injury during thyroid surgery) is difficult to completely avoid [24-26]. Therefore, continuous IONM was introduced to monitor the state of the nerve in real-time.

Transcartilage electrodes can assess laryngeal EMG by RLN stimulation. Piezo-electric effect converts changes in pressure, acceleration, temperature, strain, or force into an electrical charge. Pressure sensor using piezo-electric effect can convert pressure on the surface to an electrical charge. Thus, it can measure the changes of laryngeal muscle twitching via RLN electrical stimulation, such as, by measuring laryngeal muscle twitching with the fingers during surgery, rather than by EMG of the vocalis muscle. One study has reported that the muscle movement by nerve stimulation could be detected using pressure sensors instead of EMG [27]. Therefore, the objectives of this study are to measure and compare the amplitude, latency, and stimulus threshold of transcartilage needle EMG to those of laryngeal twitching measured by a pressure sensor attached to an ETT after RLN electrical stimulation, and to evaluate the feasibility and reliability of a novel IONM system using an ETT with a pressure sensor. We also investigated the possibility of applying a pressure sensor to a continuous IONM system by comparing the change in amplitude between transcartilage needle EMG and laryngeal twitching measured using an ETT with a pressure sensor after RLN traction injury.

\section{MATERIALS AND METHODS}

\section{Animals and experimental setting}

This study protocol was approved by the Institutional Animal Care and Use Committee of Pusan National University Yangsan Hospital (No. PNUYH-2018-066). The experiment was conducted using eight RLNs in four Yorkshire-Landrace-Duroc female pigs $(30-40 \mathrm{~kg})$ that had undergone thyroidectomy. The anesthesia protocol was as follows: the pigs were sedated with an intramuscular injection of ketamine hydrochloride $(20 \mathrm{mg} / \mathrm{kg})$ and xylazine $(2 \mathrm{mg} / \mathrm{kg})$; anesthesia was maintained with inhaled $3 \%$ isoflurane.

All pigs were intubated with an ETT (internal diameter, $7.0 \mathrm{~mm}$ ) with an attached pressure sensor (Fig. 1). Simultaneously, transcartilage needle electrodes (Medtronic Xomed, Jacksonville, FL, USA) were inserted at the vocal cord level of both sides of the thyroid cartilage and connected to a Nerve Integrity Monitor (NIM-Response 3.0 System; Medtronic Xomed) (Fig. 2). The location of the ETT tube with pressure sensor was confirmed with a flexible fiberscope and thyroidectomy was performed. The stimulus was $100 \mu \mathrm{V}$ in duration and $4 \mathrm{~Hz}$ in frequency.

The largest amplitudes and latencies from the transcartilage needle EMG and laryngeal twitching measured by the pressure sensor attached to the ETT at eight RLNs were recorded. All pigs were monitored using an NIM III and a handmade pressure 

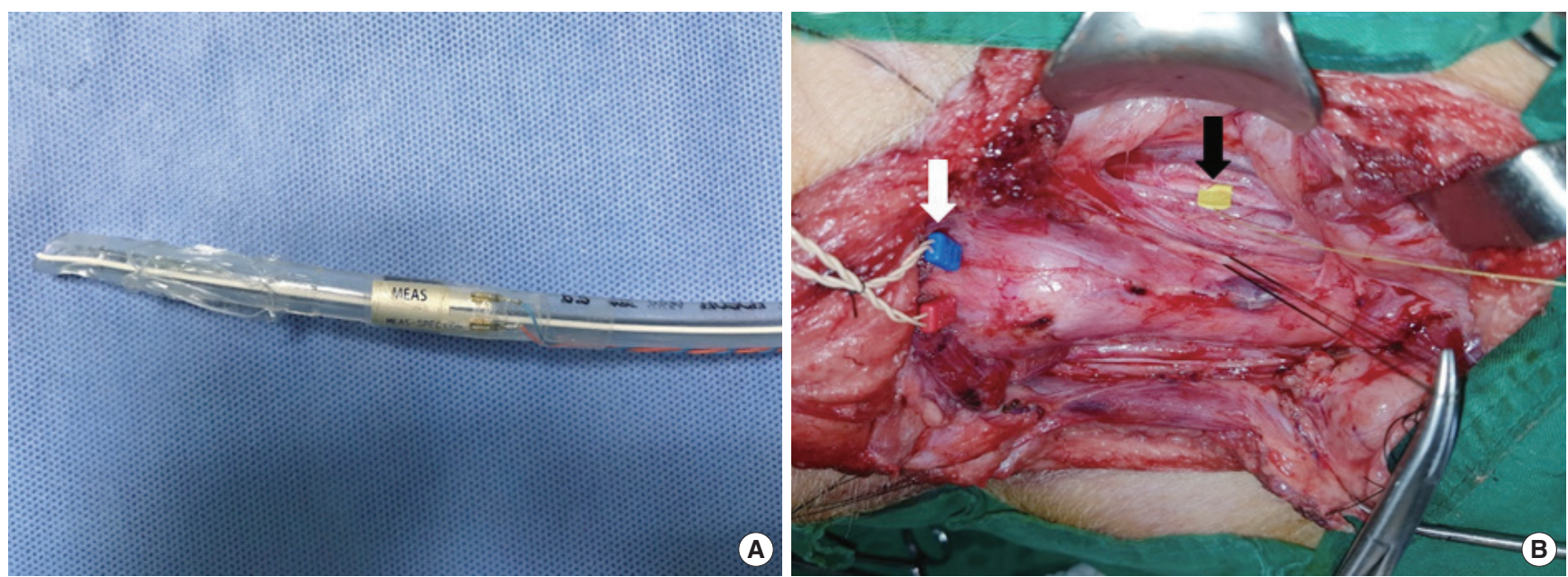

Fig. 1. (A) Novel device and surgical field. Photograph of an endotracheal tube with attached pressure sensor. (B) Photograph of the surgical field on the pig, demonstrating recurrent laryngeal nerve $(R L N)$ traction injury. Transcartilage needle electrodes were inserted into the vocal cord level of both sides of the thyroid cartilage. The automatic periodic stimulation (APS) was placed on the vagus nerve, left under continuous intermittent intraoperative neuromonitoring, and the left RLN was pulled up using silk 3-0. White arrow, electromyography electrodes; Black arrow, APS.

sensor monitoring system. At the end of the experiment, the movement of the vocal cords was confirmed by endoscopy. The pigs were euthanized after the experiment.

\section{ETT with pressure sensor}

The novel ETT with pressure sensor was developed to measure the surface pressure change on the ETT using the piezoelectric effect to detect laryngeal muscle twitching (Fig. 1A). The pressure sensor was a surface-type device attached to the ETT. Change in surface pressure on the ETT was generated by laryngeal muscle twitching due to nerve stimulation, and the pressure sensor checked the amplitude and latency of laryngeal muscle twitching. The stimulation threshold was defined as the stimulus value at which the first response appeared in EMG or in laryngeal twitching measured by the pressure sensor. We assessed the amplitude, latency, and stimulus thresholds of transcartilage needle EMG and laryngeal twitching measured by the pressure sensor in eight RLNs of four pigs.

\section{Traction injury of the RLN}

The changes in EMG and laryngeal twitching measured by the pressure sensor were compared using an experiment mimicking RLN traction injury that could be caused by medial retraction of the thyroid gland. To reproduce the RLN traction injury, the automatic periodic stimulation (APS) was placed on the vagus nerve under continuous IONM, and the ipsilateral RLN was pulled up using silk 3-0 (Fig. 1B). At the beginning of the experiment, baseline amplitudes and latencies of transcartilage needle EMG were registered. Registered stimuli were $3 \mathrm{~mA}$ during continuous IONM. When the EMG amplitude fell below $50 \%$ and the alarm sounded due to slow traction of the RLN, we stopped

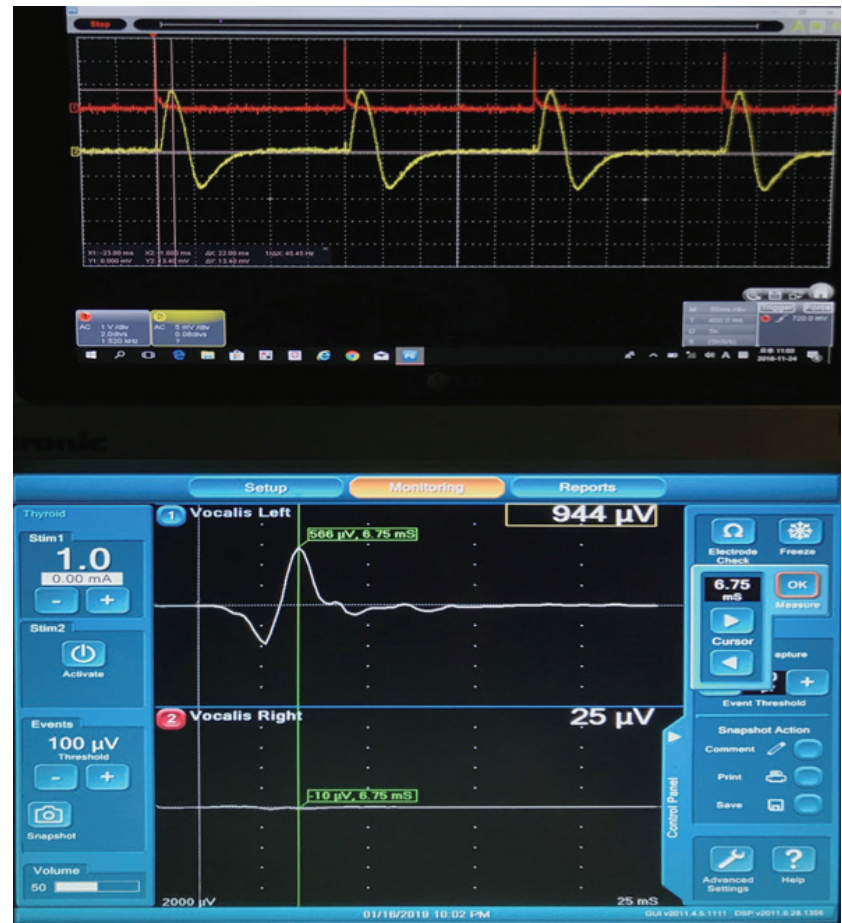

Fig. 2. Electromyography (EMG) and endotracheal tube with pressure sensor recordings, showing the amplitude and latency of EMG and the pressure sensor for $1 \mathrm{~mA}$ stimulation using the Nerve Integrity Monitor 3.0 system in pig. Upper graph, pressure sensor signal; Lower graph, EMG signal.

the RLN traction and observed the change in the amplitude of EMG and that of laryngeal twitching for 20 minutes. We measured the change in the amplitudes of EMG and laryngeal 
twitching measured by the pressure sensor, in real-time under continuous IONM.

\section{Statistical analysis}

Statistical analyses were performed using the Wilcoxon signedrank test with IBM SPSS ver. 21.0 (IBM Corp., Armonk, NY, USA). A $P$-value less than 0.05 was considered a statistically significant difference.

\section{RESULTS}

\section{Comparison between EMG and pressure sensor}

The stimulation thresholds, amplitudes, and latencies of EMG and laryngeal twitching measured by pressure sensor were investigated (Table 1, Fig. 2). The ETT with pressure sensor was feasible and reliable for use in this experiment. The mean amplitudes of EMG and laryngeal twitching measured by pressure sensor were $1,073.09 \pm 477.28 \mu \mathrm{V}$ and 5,808.92 $\pm 3,319.21 \mu \mathrm{V}$ respectively, and the mean latencies were $4.63 \pm 1.45 \mathrm{~ms}$ and $19.63 \pm 2.19 \mathrm{~ms}$, respectively. The amplitude and latency differed significantly between EMG and the pressure sensor $(P<0.001$, $P<0.001$, respectively). Both EMG and the pressure sensor showed a response to $0.3 \mathrm{~mA}$ stimulation in all RLNs. There were no differences in the stimulation threshold to evaluate the RLN status between EMG and the pressure sensor.

\section{Changes in amplitudes of EMG and pressure sensor depending on stimulation}

As the stimulus intensity increased from $0.3 \mathrm{~mA}$, the EMG amplitude gradually increased. However, the amplitude did not increase on stimulation above $1.0 \mathrm{~mA}$ and plateaued. Similar to EMG, the amplitude of the pressure sensor measuring laryngeal muscle twitching increased with increasing stimulus by $0.3 \mathrm{~mA}$ or more but did not increase further with amplitude above 1.0 $\mathrm{mA}$ and plateaued (Fig. 3).

\section{Traction injury of the RLN}

After RLN traction injury, the amplitudes of EMG and the pressure sensor attached to the ETT under continuous IONM were investigated in eight RLNs. The mean amplitudes of EMG at the baseline before RLN traction, when RLN traction stopped, and

Table 1. Analysis of the amplitude and latency of transcartilage needle EMG and ETT with pressure sensor depending on the value of the RLN stimulus under IONM in pigs

\begin{tabular}{|c|c|c|c|c|c|c|c|c|}
\hline \multirow{2}{*}{ Stimulus (mA) } & \multicolumn{3}{|c|}{ Transcartilage needle EMG } & \multicolumn{3}{|c|}{ ETT with pressure sensor } & \multicolumn{2}{|c|}{$P$-value } \\
\hline & $\mathrm{n}$ & Amplitude $(\mu \mathrm{V})$ & Latency (ms) & $\mathrm{n}$ & Amplitude $(\mu \mathrm{V})$ & Latency (ms) & Amplitude & Latency \\
\hline 0.3 & 8 & $786.88 \pm 533.04$ & $4.65 \pm 1.52$ & 8 & $4,230.50 \pm 3,564.88$ & $19.63 \pm 2.32$ & $<0.001$ & $<0.001$ \\
\hline 0.5 & 8 & $1,051.00 \pm 500.29$ & $4.62 \pm 1.55$ & 8 & $5,312.50 \pm 3,314.65$ & $19.63 \pm 2.32$ & $<0.001$ & $<0.001$ \\
\hline 0.8 & 8 & $1,134.38 \pm 455.99$ & $4.62 \pm 1.55$ & 8 & $5,862.50 \pm 3,539.95$ & $19.63 \pm 2.32$ & $<0.001$ & $<0.001$ \\
\hline 1.0 & 8 & $1,152.88 \pm 489.19$ & $4.65 \pm 1.52$ & 8 & $6,187.50 \pm 3,444.84$ & $19.63 \pm 2.32$ & $<0.001$ & $<0.001$ \\
\hline 1.5 & 8 & $1,124.38 \pm 485.01$ & $4.62 \pm 1.55$ & 8 & $6,400.00 \pm 3,570.51$ & $19.63 \pm 2.32$ & $<0.001$ & $<0.001$ \\
\hline 2.0 & 8 & $1,120.63 \pm 469.80$ & $4.62 \pm 1.55$ & 8 & $6,300.00 \pm 3,266.27$ & $19.63 \pm 2.32$ & $<0.001$ & $<0.001$ \\
\hline 3.0 & 8 & $1,141.50 \pm 485.30$ & $4.62 \pm 1.55$ & 8 & $6,362.50 \pm 3,279.78$ & $19.63 \pm 2.32$ & $<0.001$ & $<0.001$ \\
\hline Average & & $1,073.09 \pm 477.28$ & $4.63 \pm 1.45$ & & $5,808.92 \pm 3,319.21$ & $19.63 \pm 2.19$ & $<0.001$ & $<0.001$ \\
\hline
\end{tabular}

Values are presented as mean \pm SD. $P$-value indicates the result of the comparison test for the mean response values of the amplitude and latency between transcartilage needle EMG and ETT with pressure sensor depending on stimulus value.

EMG, electromyography; ETT, endotracheal tube; RLN, recurrent laryngeal nerve; IONM, intermittent intraoperative neuromonitoring; SD, standard deviation.
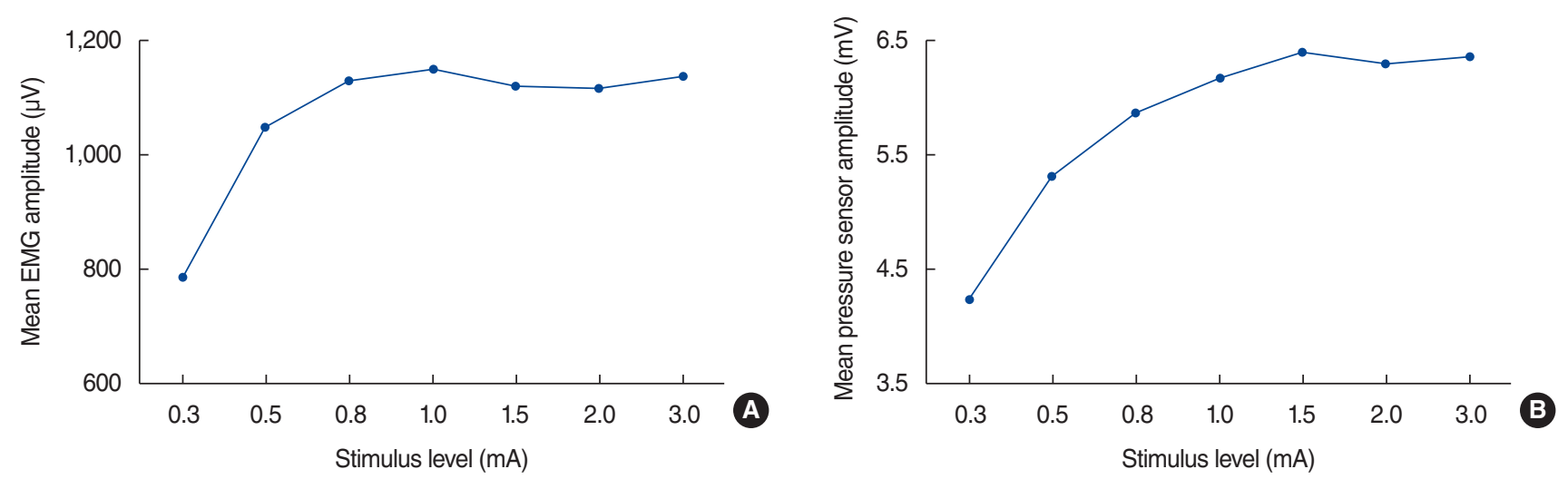

Fig. 3. Change in electromyography (EMG) and pressure sensor according to stimulus. The amplitudes of EMG (A) and the pressure sensor (B) of the vocalis muscle depending on stimulus level. 
20 minutes after stopping RLN traction were $477.50 \pm 85.64 \mu \mathrm{V}$, $199.63 \pm 89.35 \mu \mathrm{V}(40.9 \%$ of baseline, $P<0.001)$, and $267.63 \pm$ $90.51 \mu \mathrm{V}(56.1 \%$ of baseline, $P<0.001)$, respectively (Table 2 , Figs. 4 and 5). However, it still did not recover 20 minutes after stopping RLN traction (Supplementary Material 1, Video Clip 1). The mean latency time of EMG was $8.58 \pm 2.55 \mathrm{~ms}$ before RLN traction, when RLN traction stopped, and 20 minutes after stop- ping RLN traction. The mean amplitudes of the pressure sensor at the baseline before RLN traction, when RLN traction stopped, and 20 minutes after stopping RLN traction were 5,837.50土 $3,185.65 \mu \mathrm{V}, 3,112.50 \pm 2,010.99 \mu \mathrm{V}(54.6 \%$ of baseline, $P<$ $0.001)$, and $5,112.50 \pm 3,162.02 \mu \mathrm{V}(87 \%$ of baseline, $P=0.221)$, respectively (Table 2, Figs. 4 and 5). It almost recovered (Supplementary Material 1, Video Clip 1). The mean latency time of the

Table 2. Analysis of the mean amplitude and latency of the transcartilage needle EMG and ETT with pressure sensor depending on the time of the RLN traction injury under continuous IONM in pigs

\begin{tabular}{|c|c|c|c|c|}
\hline \multirow{2}{*}{ Variable } & \multicolumn{3}{|c|}{ Time (3 mA) } & \multirow{2}{*}{$P$-value } \\
\hline & Before $(n=8)$ & Stop $(n=8)$ & After $(n=8)$ & \\
\hline \multicolumn{5}{|c|}{ Transcartilage needle EMG } \\
\hline Amplitude $(\mu \mathrm{V})$ & $477.50 \pm 85.64(100)$ & $199.63 \pm 89.35(40.88)$ & $267.63 \pm 90.51(56.13)$ & $\begin{array}{r}<0.001^{a)} \\
0.044^{b)} \\
<0.001^{c)}\end{array}$ \\
\hline Latency (ms) & $8.58 \pm 2.55$ & $8.58 \pm 2.55$ & $8.58 \pm 2.55$ & \\
\hline \multicolumn{5}{|c|}{ ETT with pressure sensor } \\
\hline Amplitude $(\mu \mathrm{V})$ & $5,837.50 \pm 3,185.65(100)$ & $3,112.50 \pm 2,010.99(54.63)$ & $5,112.50 \pm 3,162.02(87)$ & $\begin{array}{r}<0.001^{a)} \\
0.001^{b)} \\
0.221^{c)}\end{array}$ \\
\hline Latency (ms) & $23.88 \pm 3.56$ & $24.00 \pm 3.38$ & $23.88 \pm 3.56$ & \\
\hline
\end{tabular}

Values are presented as mean $\pm \mathrm{SD}(\%)$ or mean $\pm \mathrm{SD}$.

EMG, electromyography; ETT, endotracheal tube; RLN, recurrent laryngeal nerve; IONM, intermittent intraoperative neuromonitoring; Before, before RLN traction; Stop, when RLN traction stopped; After, 20 minutes after RLN traction; SD, standard deviation.

a) $P$-value between before RLN traction and when RLN traction stopped. ${ }^{b)} P$-value between when RLN traction stopped and 20 minutes after RLN traction. c) $P$ value between Before RLN traction and 20 minutes after RLN traction.
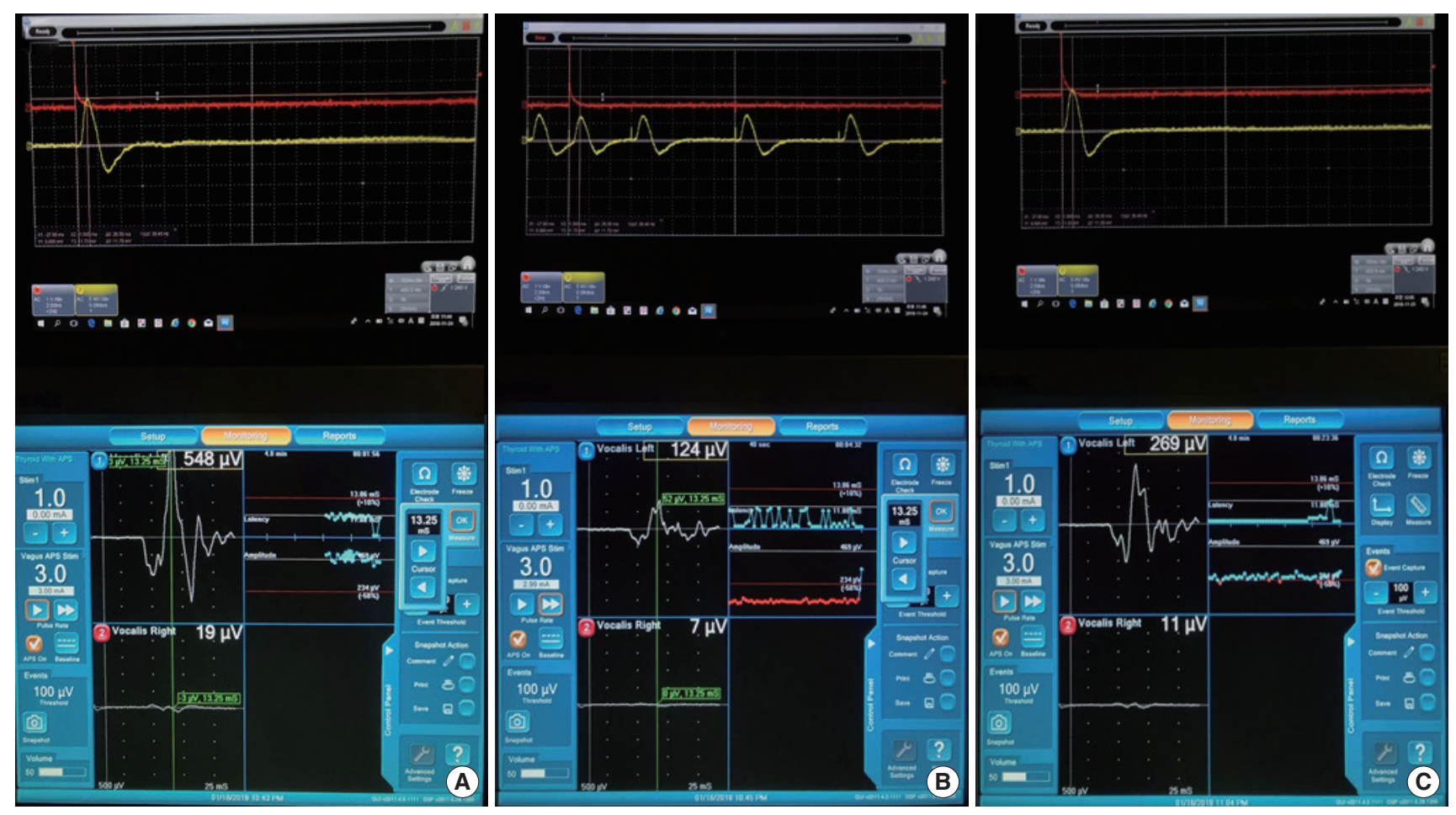

Fig. 4. Continuous amplitude changes of electromyography (EMG) and pressure sensor before recurrent laryngeal nerve (RLN) traction (A), when RLN traction stopped (B), and 20 minutes after RLN traction (C) for 3-mA stimulation using the Nerve Integrity Monitor 3.0 system in pigs. Upper graph, pressure sensor signal; Lower graph, EMG signal. 


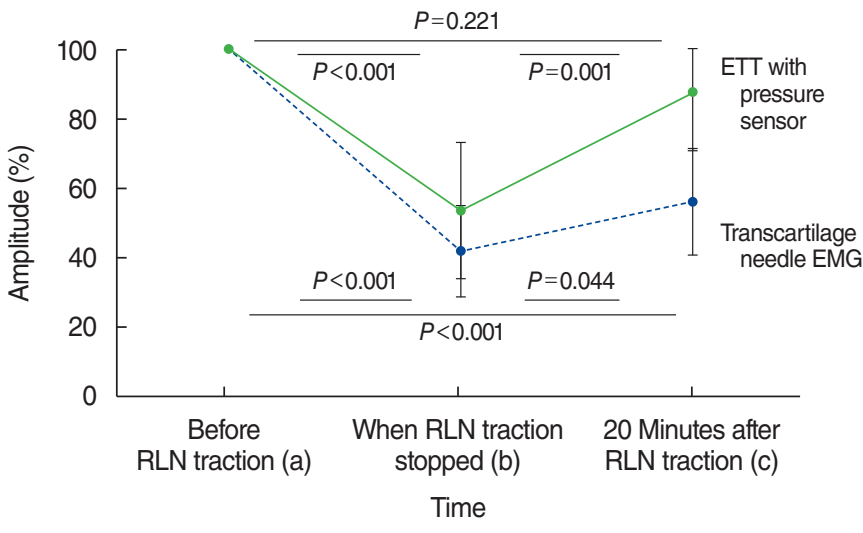

Fig. 5. Amplitude (\%) patterns of electromyography (EMG, dotted line) and the pressure sensor (solid line) change depending on the time of the recurrent laryngeal nerve (RLN) traction injury: before RLN traction (a), when $R L N$ traction stopped (b), and 20 minutes after $R L N$ traction (c). a vs. b: $P<0.001$, b vs. c: $P=0.001$, a vs. c: $P=0.221$ in pressure sensor; a vs. b: $P<0.001$, b vs. c: $P=0.044$, a vs. c: $P<0.001$ in EMG. ETT, endotracheal tube.

pressure sensor were $23.88 \pm 3.56 \mathrm{~ms}$ before RLN traction, $24.00 \pm 3.38 \mathrm{~ms}$ when RLN traction stopped, and $23.88 \pm 3.56$ ms 20 minutes after stopping RLN traction. Fig. 5 presents the changes in amplitudes of EMG and the pressure sensor depending on the time of measurement. After the end of the experiment, vocal cord movement was confirmed by endoscopy before pigs were euthanized. Bilateral vocal fold movement was observed in all four pigs.

\section{DISCUSSION}

In this study, there were significant differences in values of amplitude and latency time between EMG and pressure sensor, however no difference in stimulus threshold. The experiment of RLN traction injury has shown that the pressure sensor reflects the condition of the RLN well. The use of IONM during thyroid surgery has been a worldwide phenomenon [16]. However, there were many reports that the use of IONM during thyroid surgery did not help to reduce the permanentVFP ratio [16,28-33]. Therefore, clinicians face a dilemma regarding using IONM when considering the costs. However, a recent meta-analysis has documented that IONM may reduce the transient and persistent VFP ratios [34].

Although IONM helps prevent VFP, there are disadvantages to using it. Conventional EMG using EMG surface electrodes may cause very low signal or LOS due to malposition of the tube $[7,15,24]$. This means that the contact between the EMG surface electrodes and the vocal cords is likely to change due to cervical extension or surgical manipulation $[14,24,25]$. Contact between the vocalis muscle and the electrode is critical to secure an exact IONM signal. As previously mentioned, there have been many attempts to overcome the disadvantages of EMG [35]. However, many methods use EMG to evaluate nerve status and require that two EMG electrodes be attached or inserted into the larynx.

Therefore, we sought to test a means to measure laryngeal muscle twitching with a pressure sensor. A previous study has reported that the pressure sensor has the same stimulus threshold as EMG in rabbits [27]. An accelerometer sensor placed in anterior neck skin and the posterior cricoid also has the same stimulus threshold as EMG in pigs [36]. These reports have suggested that the accuracy of the pressure sensor to identify laryngeal muscle twitching to assess RLN status is likely to be very similar to that of EMG. In addition, it is also expected that when a pressure sensor has only partial contact with the surface of the vocal cords, laryngeal twitching may still be detected, compared to the EMG method in which two electrodes are attached to the vocal cords. Even if the sensor part does not come into contact with the vocal cords, the pressure change generated by the movement of the vocal cords is transferred to the ETT and then transferred to the sensor, detecting the change in the vocal cords movement. In the present study, there were no differences in the stimulus threshold between EMG and pressure sensor.

As shown in Fig. 3, the amplitude of EMG started at $0.3 \mathrm{~mA}$, and the amplitude increased with increasing stimulus intensity. When the stimulus was more than $1.0 \mathrm{~mA}$, the amplitude showed a plateau. The change in amplitude due to the stimulation of the pressure sensor showed a pattern similar to EMG. Randolph et al. [37] showed that the amplitude of EMG increased with increasing stimulus from $0.4 \mathrm{~mA}$, but the amplitude was almost at a plateau from stimulation above $0.8 \mathrm{~mA}$. In other words, there is a positive correlation between laryngeal EMG amplitude and laryngeal palpation rating.

When a traction injury to the RLN occurs, the amplitude of EMG decreases. Continuous IONM was developed to prevent trauma-induced nerve damage by sending an alarm signal when the EMG amplitude is reduced by more than $50 \%$. There were no reports about comparisons between EMG amplitude and laryngeal muscle twitching using a pressure sensor after RLN traction injury. In this study, we measured the amplitude of laryngeal muscle twitching as checked by a pressure sensor after RLN traction. The amplitudes and latencies of the pressure sensor and EMG were compared. In RLN traction injury experiments, the EMG amplitude still did not recover 20 minutes after stopping RLN traction. However, the amplitude of the pressure sensor almost recovered.

Generally, when a traction injury occurs, if the amplitude is reduced by $50 \%$ or more, nerve damage is common. If the amplitude does not recover to near-normal, preservation of RLN is not guaranteed. Changes in the pressure sensor during traction injury are similar to those of the EMG pattern, but the amplitude is slightly less reduced and the recovery is better. Since the movement of all pigs' vocal cords were confirmed after the experiment, the pressure sensor reflects the condition of the laryngeal muscle twitching. In the future, it is expected that continu- 
ous IONM development will be possible using pressure sensors to measure laryngeal twitching.

There are some limitations to this study. First, the amplitude of the pressure sensor was very high and distinct from that of the EMG. However, the gap in amplitude was due to discrepancies in the measurement method. Therefore, the amplitude difference between EMG and pressure sensor is not meaningful. Second, this study was a small animal experiment. To demonstrate the feasibility and safety of this new device, large-scale human studies are needed.

In conclusion, the ETT with pressure sensor was able to identify laryngeal twitching. The change in amplitude due to stimulation of the pressure sensor showed a pattern similar to EMG. Pressure sensors can be used for RLN traction injury prediction as well as RLN identification and preservation through muscle twitching. Therefore, the pressure sensor is feasible and reliable to detect laryngeal twitching. Our novel IONM system using an ETT with pressure sensor can be an alternative to EMG.

\section{CONFLICT OF INTEREST}

No potential conflict of interest relevant to this article was reported.

\section{ORCID}

$\begin{array}{ll}\text { Eui-Suk Sung } & \text { https://orcid.org/0000-0001-8752-3426 } \\ \text { Sung-Chan Shin } & \text { https://orcid.org/0000-0003-2329-0648 } \\ \text { Hyun-Keun Kwon } & \text { https://orcid.org/0000-0003-4089-8639 } \\ \text { Jia Kim } & \text { https://orcid.org/0000-0001-9797-5135 } \\ \text { Da-Hee Park } & \text { https://orcid.org/0000-0001-7466-2793 } \\ \text { Seong-Wook Choi } & \text { https://orcid.org/0000-0002-2459-462X } \\ \text { Sang-Hoon Kim } & \text { https://orcid.org/0000-0001-6186-0790 } \\ \text { Jin-Choon Lee } & \text { https://orcid.org/0000-0002-5629-4277 } \\ \text { Jung-Hoon Ro } & \text { https://orcid.org/0000-0002-5203-5956 } \\ \text { Byung-Joo Lee } & \text { https://orcid.org/0000-0001-7091-6688 }\end{array}$

\section{AUTHOR CONTRIBUTIONS}

Conceptualization: BJL. Data curation: JK, DHP, SWC, SHK. Formal analysis: SCS, HGK. Methodology: JHR. Project administration: JCL. Visualization: JHR. Writing-original draft: ESS. Writing-review \& editing: ESS, BJL.

\section{SUPPLEMENTARY MATERIALS}

Supplementary materials can be found via https://doi.org/10. 21053/ceo.2019.01249.

\section{REFERENCES}

1. Haugen BR, Alexander EK, Bible KC, Doherty GM, Mandel SJ, Nikiforov YE, et al. 2015 American Thyroid Association Management Guidelines for Adult Patients with Thyroid Nodules and Differentiated Thyroid Cancer: The American Thyroid Association Guidelines Task Force on Thyroid Nodules and Differentiated Thyroid Cancer. Thyroid. 2016 Jan;26(1):1-133.

2. Chandrasekhar SS, Randolph GW, Seidman MD, Rosenfeld RM,Angelos P, Barkmeier-Kraemer J, et al. Clinical practice guideline: improving voice outcomes after thyroid surgery. Otolaryngol Head Neck Surg. 2013 Jun;148(6 Suppl):S1-37.

3. Jeannon JP, Orabi AA, Bruch GA,Abdalsalam HA, Simo R. Diagnosis of recurrent laryngeal nerve palsy after thyroidectomy: a systematic review. Int J Clin Pract. 2009 Apr;63(4):624-9.

4. RiddellV.Thyroidectomy: prevention of bilateral recurrent nerve palsy. Results of identification of the nerve over 23 consecutive years (1946-69) with a description of an additional safety measure. Br J Surg. 1970 Jan;57(1):1-11.

5. Hermann M,Alk G, Roka R, Glaser K, Freissmuth M. Laryngeal recurrent nerve injury in surgery for benign thyroid diseases: effect of nerve dissection and impact of individual surgeon in more than 27,000 nerves at risk. Ann Surg. 2002 Feb;235(2):261-8.

6. Chiang FY,Wang LF, Huang YF, Lee KW, KuoWR. Recurrent laryngeal nerve palsy after thyroidectomy with routine identification of the recurrent laryngeal nerve. Surgery. 2005 Mar;137(3):342-7.

7. Randolph GW, Dralle H; International Intraoperative Monitoring Study Group, Abdullah H, Barczynski M, Bellantone R, et al. Electrophysiologic recurrent laryngeal nerve monitoring during thyroid and parathyroid surgery: international standards guideline statement. Laryngoscope. 2011 Jan;121 Suppl 1:S1-16.

8. Sung ES, Lee JC, Shin SC, Choi SW, Jung DW, Lee BJ. Development of a novel detachable magnetic nerve stimulator for intraoperative neuromonitoring. World J Surg. 2018 Jan;42(1):137-42.

9. Sung ES, Lee JC, Kim SH, Shin SC, Jung DW, Lee BJ. Development of an attachable endoscopic nerve stimulator for intraoperative neuromonitoring during endoscopic or robotic thyroidectomy. Otolaryngol Head Neck Surg. 2018 Mar;158(3):465-8.

10. Shin SC, Sung ES, Choi SW, Kim SD, Jung DW, Kim SH, et al. Feasibility and safety of nerve stimulator attachment to energy-based devices: a porcine model study. Int J Surg. 2017 Dec;48:155-9.

11. Snyder SK, Hendricks JC. Intraoperative neurophysiology testing of the recurrent laryngeal nerve: plaudits and pitfalls. Surgery. 2005 Dec; 138(6):1183-91.

12. Beldi G, Kinsbergen T, Schlumpf R. Evaluation of intraoperative recurrent nerve monitoring in thyroid surgery. World J Surg. 2004 Jun; 28(6):589-91.

13. Lu IC, Chu KS, Tsai CJ, Wu CW, Kuo WR, Chen HY, et al. Optimal depth of NIM EMG endotracheal tube for intraoperative neuromonitoring of the recurrent laryngeal nerve during thyroidectomy. World J Surg. 2008 Sep;32(9):1935-9.

14. Wu CW, Wang MH, Chen CC, Chen HC, Chen HY, Yu JY, et al. Loss of signal in recurrent nerve neuromonitoring: causes and management. Gland Surg. 2015 Feb;4(1):19-26.

15. Dionigi G, Bacuzzi A, Boni L, Rovera F, Dionigi R. What is the learning curve for intraoperative neuromonitoring in thyroid surgery? Int J Surg. 2008;6 Suppl 1:S7-12.

16. Dralle H, Sekulla C, Lorenz K, Brauckhoff M, Machens A; German IONM Study Group. Intraoperative monitoring of the recurrent laryngeal nerve in thyroid surgery. World J Surg. 2008 Jul;32(7):1358-66.

17. Khan A, Pearlman RC, Bianchi DA, Hauck KW. Experience with two types of electromyography monitoring electrodes during thyroid surgery.Am J Otolaryngol. 1997 Mar-Apr;18(2):99-102. 
18. Tschopp KP, Gottardo C. Comparison of various methods of electromyographic monitoring of the recurrent laryngeal nerve in thyroid surgery. Ann Otol Rhinol Laryngol. 2002 Sep;111(9):811-6.

19. Rea JL, Khan A. Clinical evoked electromyography for recurrent laryngeal nerve preservation: use of an endotracheal tube electrode and a postcricoid surface electrode. Laryngoscope. 1998 Sep;108(9): 1418-20.

20. Marcus B, Edwards B, Yoo S, Byrne A, Gupta A, Kandrevas J, et al. Recurrent laryngeal nerve monitoring in thyroid and parathyroid surgery: the University of Michigan experience. Laryngoscope. 2003 Feb;113(2):356-61.

21. Liddy W, Barber SR, Lin BM, Kamani D, Kyriazidis N, Lawson B, et al. Monitoring of the posterior cricoarytenoid muscle represents another option for neural monitoring during thyroid surgery: normative vagal and recurrent laryngeal nerve posterior cricoarytenoid muscle electromyographic data. Laryngoscope. 2018 Jan;128(1):283-9.

22. Wu CW, Chiang FY, Randolph GW, Dionigi G, Kim HY, Lin YC, et al. Feasibility of intraoperative neuromonitoring during thyroid surgery using transcartilage surface recording electrodes. Thyroid. 2018 Nov; 28(11):1508-16.

23. Wu CW, Chiang FY, Randolph GW, Dionigi G, Kim HY, Lin YC, et al. Transcutaneous recording during intraoperative neuromonitoring in thyroid surgery. Thyroid. 2018 Nov;28(11):1500-7.

24. Chiang FY, Lu IC, KuoWR, Lee KW, Chang NC, Wu CW.The mechanism of recurrent laryngeal nerve injury during thyroid surgery: the application of intraoperative neuromonitoring. Surgery. 2008 Jun; 143(6):743-9.

25. Chiang FY, Lee KW, Chen HC, Chen HY, Lu IC, KuoWR, et al. Standardization of intraoperative neuromonitoring of recurrent laryngeal nerve in thyroid operation. World J Surg. 2010 Feb;34(2):223-9.

26. Schneider R, Randolph GW, Sekulla C, Phelan E, Thanh PN, Bucher $\mathrm{M}$, et al. Continuous intraoperative vagus nerve stimulation for identification of imminent recurrent laryngeal nerve injury. Head Neck. 2013 Nov;35(11):1591-8.

27. Sung ES, Lee JC, Shin SC, Kwon HG, Kim MS, Kim DJ, et al. Development of a novel intraoperative neuromonitoring system using a surface pressure sensor to detect muscle movement: a rabbit model study. Clin Exp Otorhinolaryngol. 2019 May;12(2):217-23.

28. Sanabria A, Silver CE, Suarez C, Shaha A, Khafif A, Owen RP, et al. Neuromonitoring of the laryngeal nerves in thyroid surgery: a critical appraisal of the literature. Eur Arch Otorhinolaryngol. 2013 Sep; 270(9):2383-95.

29. Lifante JC, McGill J, MurryT, Aviv JE, InabnetWB 3rd.A prospective, randomized trial of nerve monitoring of the external branch of the superior laryngeal nerve during thyroidectomy under local/regional anesthesia and IV sedation. Surgery. 2009 Dec;146(6):1167-73.

30. Barczynski M, Konturek A, Cichon S. Randomized clinical trial of visualization versus neuromonitoring of recurrent laryngeal nerves during thyroidectomy. Br J Surg. 2009 Mar;96(3):240-6.

31. Higgins TS, Gupta R, Ketcham AS, Sataloff RT, Wadsworth JT, Sinacori JT. Recurrent laryngeal nerve monitoring versus identification alone on post-thyroidectomy true vocal fold palsy: a meta-analysis. Laryngoscope. 2011 May;121(5):1009-17.

32. Dionigi G, Boni L, Rovera F, Bacuzzi A, Dionigi R. Neuromonitoring and video-assisted thyroidectomy: a prospective, randomized casecontrol evaluation. Surg Endosc. 2009 May;23(5):996-1003.

33. Chan WF, Lo CY. Pitfalls of intraoperative neuromonitoring for predicting postoperative recurrent laryngeal nerve function during thyroidectomy.World J Surg. 2006 May;30(5):806-12.

34. Bai B, Chen W. Protective effects of intraoperative nerve monitoring (IONM) for recurrent laryngeal nerve injury in thyroidectomy: meta-analysis. Sci Rep. 2018 May;8(1):7761.

35. Chiang FY, Lu IC, Chang PY, Dionigi G, Randolph GW, Sun H, et al. Comparison of EMG signals recorded by surface electrodes on endotracheal tube and thyroid cartilage during monitored thyroidectomy. Kaohsiung J Med Sci. 2017 Oct;33(10):503-9.

36. Sung ES, Lee JC, Shin SC, Kwon HK, Na HS, Park DH, et al. Development of a novel intraoperative neuromonitoring system using an accelerometer sensor in thyroid surgery: a porcine model study. Clin Exp Otorhinolaryngol. 2019 Nov;12(4):420-6.

37. Randolph GW, Kobler JB, Wilkins J. Recurrent laryngeal nerve identification and assessment during thyroid surgery: laryngeal palpation. World J Surg. 2004 Aug;28(8):755-60. 